\title{
Infections: The Achilles Heel of Immunosuppression for Renal Transplantation and Immunological Renal Disease Management in the Top End of Northern Australia: Case Reports*
}

\author{
Sandawana William Majoni ${ }^{1,2}$, Kerry Dole ${ }^{1}$, Zulfikar Jabbar ${ }^{1,2}$, Madhivanan Sundram ${ }^{1}$, Greg Perry ${ }^{1,2}$ \\ ${ }^{1}$ Department of Nephrology, Royal Darwin Hospital, Division of Medicine, Casuarina, Australia \\ ${ }^{2}$ Flinders University and Northern Territory Clinical School, Royal Darwin Hospital Campus, Tiwi, Australia \\ Email: sandawanaw@aol.com
}

Received September 18, 2012; revised October 24, 2012; accepted November 3, 2012

\begin{abstract}
Improved immunosuppression regimens have led to better survival for patients with renal transplant grafts and patients with immunological renal diseases worldwide. However, this is not the case in the Northern Territory of Australia. Available limited published data from the Northern Territory of Australia have shown poor outcomes for renal transplantation with survival for both patients and grafts around $50 \%$ at 5 years suggesting death with a functioning graft as the commonest cause of graft loss. These studies have shown that the leading cause of death is infections. Achieving the right level of immunosuppression to prevent rejection in renal transplantation and achieve remission in immunological renal diseases can be a major challenge in areas with high prevalence of infections such as the Northern Territory. We present 2 cases of the challenges from infections of immunosuppression in renal transplantation and immunological renal diseases in the Northern Territory of Australia. A 57-year-old Aboriginal woman received a deceased donor renal transplant in 2006. She has been plagued by recurrence of several life threatening infections including urinary tract, cytomegalovirus, and severe cryptococcocus infections. This resulted in immunosuppression reduction and failure of the transplant 5 years post transplantation. A 20-year-old Aboriginal woman presented with a combination of severe lupus nephritis and severe sepsis. She fully recovered after treatment with antibiotics and careful immunosuppression. However, she has had recurrent hospital admissions with life threatening infections resulting in stopping the immunosuppression. She then had severe lupus nephritis flare leading to dialysis dependence and will need a renal transplant. The cases illustrate the need for tailored and robust immunosuppression and transplant work up protocols. To that effect, prospective studies to analyse outcomes in immunosuppressed individuals, pharmacokinetic studies assessing whether the conventionally recommended drug levels are appropriate for this population and culturally appropriate educational programmes need to be performed.
\end{abstract}

Keywords: Renal Transplant; Immunological Renal Disease; Lupus Nephritis; Immunosuppression; Indigenous Australians; Northern Territory

\section{Introduction}

Data from the Australia and New Zealand Dialysis and Transplant Registry (ANZDATA) and other studies show that the prevalence and incidence of chronic kidney disease are 8 - 10 times higher in Indigenous Australians of the Northern Territory than non-indigenous population groups in Australia. Prevalent rates of dialysis requirements of $700-1200$ per million population have been shown [1,2]. Indigenous patients with chronic kidney disease are 10 to 20 years younger than their Non-Indigenous counterparts. Renal transplantation is the best

*Competing interests: The authors declare that they have no competing interests treatment for end stage renal disease.

Rates of renal transplantation are lower in this group because of limited availability of compatible donors, the reduced chances of getting on the deceased donor waiting list $[3,4]$, very high cardiovascular and other comorbidities pre-transplantation and the high risk to the donors associated with living renal transplant donation [3, 5-7]. With improved immunosuppression regimens, graft and patient survival have significantly improved over the last three decades worldwide.

However this is not the case in Indigenous Australians. The outcomes for renal transplantation as assessed in published cross sectional analyses are very poor with 
graft and patients survival both around $50 \%$ at 5 years suggesting death with functioning graft as the commonest cause of graft loss [6]. This is mainly due to infections in a population with very high incidence and prevalence of renal disease living in remote communities where transplantation would be the best mode of treatment to prevent social dislocation to urban centres for dialysis.

In areas with high risk of infections such as the Top End of the Northern Territory [8], it is challenging to determine the appropriate balance of the level of immunosuppression required to reduce the risk of rejection in transplantation and to attain disease response in patients with immunological diseases such as lupus nephritis which is also highly prevalent in this population [9-11].

We present 2 representative case reports which illustrates some of these challenges.

\section{Case Presentation}

\subsection{Case 1: A Case of Renal Transplantation}

A 57-year-old Aboriginal woman first presented with end stage renal failure to the unit in 2003 when she was started on haemodialysis via a left brachiocephalic arteriovenous fistula. The cause of her renal failure was presumed diabetic nephropathy. She had to move away from her remote community to live in the city where dialysis was available. Other commodities included type 2 diabetes mellitus, hypertension, controlled rheumatic heart disease and ischaemic heart disease.

She received a deceased donor five antigen mismatch renal transplant in 2006. The immunosuppression consisted of tacrolimus, mycophenolate mofetil, prednisolone and basilixmab during induction and tacrolimus, mycophenolate mofetil and prednisolone for maintenance. She was Cytomegalovirus (CMV) serology positive and the donor was CMV negative so she received the full unit protocol for CMV treatment post-transplantation. She had eventful first year post transplantation with recurrent urinary tract infections and treatment for CMV viraemia eight months post transplantation with a maximum viral load of 190,000 DNA copies/ml despite having had the full treatment protocol and longer than usual period of CMV prophylaxis. This was treated with a combination of reduction of immunosuppression and valganciclovir with complete resolution. There was renal graft function impairment associated with this episode but a renal biopsy did not show any evidence of rejection or CMV cytopathy.

At the end of 4 years post transplantation, she was seen in the regular transplant clinic with unintentional progressive weight loss over a six month period. Whilst undergoing investigations for this weight loss, she was admitted to hospital with diarrhoea, poor appetite and a fever of 38 degrees Celsius. Her renal allograft function was impaired due to dehydration. The diarrhoea stopped with fluid resuscitation with improvement in the renal transplant function.

Two days post admission, she developed sudden onset of confusion. She was found to have Cryptococcus meningitis and disseminated Cryptococcus disease. She also had an Escherichia coli septicaemia and urinary tract infection and required prolonged admission in Intensive Care unit for treatment. She was also found to have BK Polyomavirus viraemia with maximum viral load of 1300 Copies/ml. The CMV viraemia also recurred at the same time with viral loads of up 6580 Copies/ml. Immunosuppression was reduced to prednisolone only.

She recovered and underwent a prolonged period of rehabilitation at home after discharged from hospital. She recovered from the severe infections but her graft was failing. She had a renal biopsy which confirmed transplant glomerulopathy and acute rejection with no evidence of BK Polyomavirus or CMV cytopathy (Figures 1(a)-(c)).

Immunosuppression was escalated to dual therapy with low dose mycophenolate mofetil and prednisolone after treating the rejection with three dose pulse of intravenous methylprednisolone. Once she was stable, another renal biopsy was done which showed that the rejection process was resolving but still present so low level tacrolimus was reintroduced with trough levels being maintained around $4 \mu \mathrm{g} / \mathrm{l}$. The renal allograft function stabilized.

She was readmitted with recrudescence of life threatening urinary tract infection and septicemia and Cryptococcus meningitis three months later. She spent some time in ICU and slowly recovered. A decision was made to reduce the immunosuppression to prednisolone alone and accept the inevitable return to dialysis. She still has significant function from her renal allograft but is now dialysis dependent.

\subsection{Case 2: A Case of Immunological Renal Disease}

A 20-year-old Aboriginal woman first presented to the community health centre with a six month history of knee pain in 2009. She had very strong immunological evidence of lupus with a high homogenous pattern antinuclear antibody titre of 1:640, a high anti Double stranded DNA antibody titre of $110 \mathrm{IU} / \mathrm{ml}$ (NR $<8.0$ $\mathrm{IU} / \mathrm{ml}$ ) and complement C3 and C4 levels of $<0.40 \mathrm{~g} / \mathrm{l}$ (NR 0.83 - 2 and $<0.08$ g/L (NR 0.13 - 0.59), respectively. At that time, she had no blood or protein on urine dipstick suggestive of lupus nephritis.

Four weeks later whilst she was undergoing work up for the lupus, she was admitted to hospital as an emergency with a staphylococcal lower respiratory tract in- 


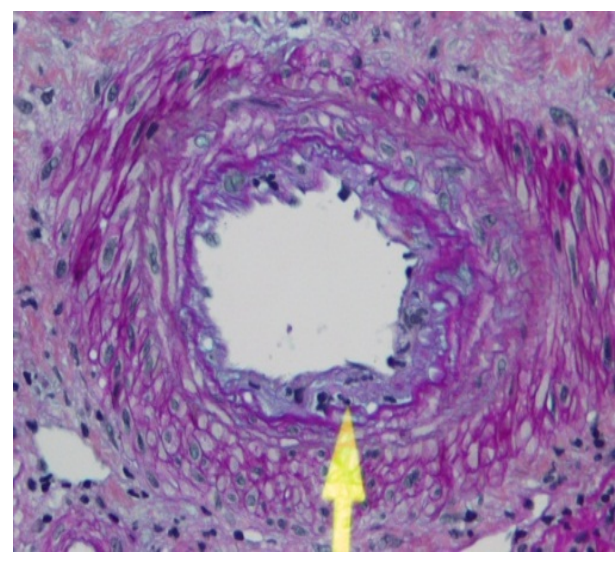

(a)

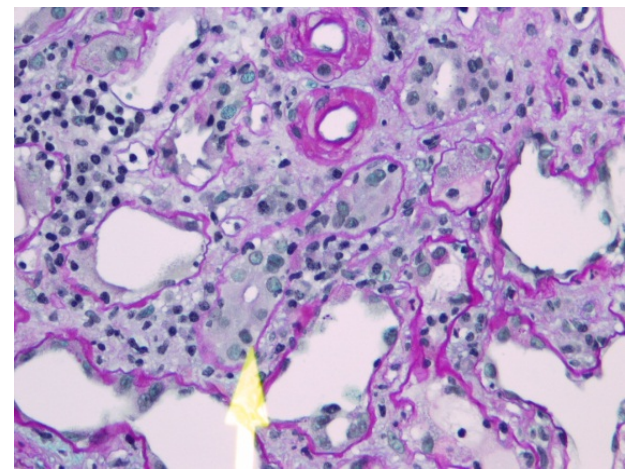

(b)

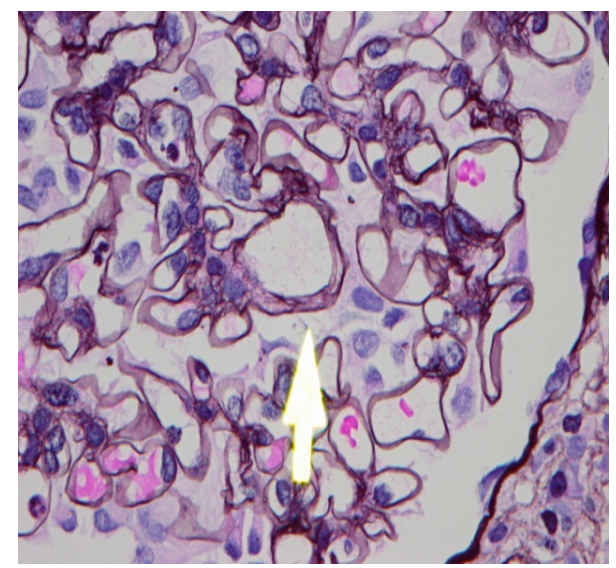

(c)

Figure 1. (a) Intimal lymphocytic infiltrates (chronic transplant arteriopathy); (b) Tubulitis (Type 2A acute cellular rejection); (c) Mesangial interposition (transplant glomerulopathy).

fection, scabies, and significant progressive renal failure. At that time, she had both glomerular haematuria and proteinuria. She was started on antibiotics and an urgent renal biopsy was done. The biopsy confirmed severe class IV lupus nephritis with 90\% crescents (Figure 2).

She presented a significant challenge as she had two potentially serious infections which would preclude the heavy immunosuppression she required. Starting her on

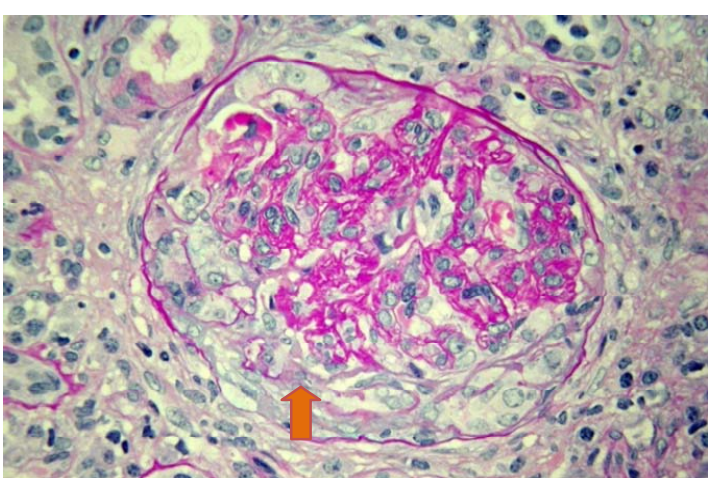

Figure 2. Class IV lupus nephritis. The biopsy showed $90 \%$ crescents (Arrow).

cyclophosphamide was considered too risky and she would not consent to it because of the risk of infertility. We concomitantly treated her with broad spectrum antibiotics, treatment for scabies and started her a mycophenolate mofetil $250 \mathrm{mg}$ twice daily together with full dose steroids. As the infections improved, we increased the dose of the mycophenolate to a maximum of $1.5 \mathrm{~g}$ twice daily.

Her renal function gradually improved and she was infection free after 4 weeks in hospital. She had a creatinine of $150 \mu \mathrm{mol} / \mathrm{l}(\mathrm{NR} 60-100 \mu \mathrm{mol} / \mathrm{l})$ when she was discharged from hospital back to her home community. She had a complicated clinical course over the following one and half years with several episodes of severe infections which included clinical cytomegalovirus colitis, severe disseminated Melioidosis, severe staphylococcal pneumonia and septicaemia complicating severe crusted scabies, streptococcal pneumonia, Herpes simplex virus infection and multiple abscesses. Her immunosuppression was stopped on all these occasions and they were all followed by deterioration of renal failure associated with increased lupus activity as evidenced by the worsening of lupus serology. Once the infections were settled, restarting the immunosuppression resulted in good response of the lupus nephritis.

She then presented at the end of 2011 with severe flare up of the lupus nephritis and end stage renal failure. She also had a combination of cerebral lupus and thrombotic microangiopathy (TMA) and a urinary tract infection. She was treated with 14 sessions of plasma exchange and antibiotics with good response of the cerebral Lupus, the TMA and the infection. She was commenced on haemodialysis and, though she has improved clinically, she remains dialysis dependent. She is still on hydroxychloquine and a small dose of prednisolone $5 \mathrm{mg}$ once daily. The challenge is how she will do if, as is being planned, she gets a kidney transplant given the complicated course she has had with immunosuppression therapy for lupus nephritis. 


\section{Discussion}

These 2 cases illustrate the challenges of managing immunosuppression in renal transplantation and immunological renal diseases in Indigenous patients in the Northern Territory of Australia. This adds to the recognised major complications associated with transplantation and immunosuppression in patients in a region with high prevalence of infections associated with social depravation and remoteness $[12,13]$. The extrapolation and universal application of results of studies of target levels of immunosuppression in different population groups to this population highlights the need to find ways of tailoring immunosuppression requirements to this patient group. This applies to other Indigenous or ethnic groups where similarly poor outcomes occur due to high levels of infections.

After the results of the cross sectional study which showed poor outcomes in renal transplantation [6], the number of those on the waiting list went down while processes were being determined to improve outcomes [4]. An immunosuppression protocol has been developed in conjunction with the infectious disease unit to improve prophylaxis and early detection of infections [8]. This has recently been updated. Though, the protocol is very robust and evidence based, it is not fully adequate because though it addresses infections such as tuberculosis, strongyloidosis, scabies, viral infections and some bacterial infections it does not include unpredictable infections such as the Cryptococcus infections as in the first case. Both patients were treated according to the protocol [8].

There is a paucity of data on the best management protocols to improve outcomes in both renal transplantation and immunological renal diseases. Work carried out recently on outcomes in transplantation has provided some insight $[6,14]$ but more prospective work including community based public health intervention needs to be assessed. Results from the impakt study have suggested some of the reasons for why patients in these regions are less likely to be transplanted but there are significant medical and socials factors contributing to the challenges on improving post transplantation outcomes and outcomes in immunological renal disease particularly in relation to the very high risk of infections [15-20].

Cases such as this and many others should lead to improvement in the immunosuppression and transplant work up process. A database to prospectively follow up all immunosuppressed patients should be developed. A pharmacokinetic study to determine the appropriate level of immunosuppression in this patient group needs to be carried out. Though the level of immunological incomepatibility with donors may be higher for renal transplant recipients, the level of immunosuppression may not necessarily need to be the same as conventionally deter- mined in other population groups. Extensive culturally relevant educational programmes need to be carried out to improve patient understanding of the benefits and risks of immunosuppression in both transplant recipients and those with immunological renal diseases.

Published experience in dealing with these complex issues is limited and more work needs to be done to improve the scope of knowledge in this area. Most of the limited data are cross sectional and retrospective analyses so, prospective cohort and interventional studies would be warranted to improve outcomes in this vulnerable group.

\section{Author Contributions}

SWM collected the data, analysed the information and wrote the first draft of the manuscript. KD collected the data. ZJ, MS and GP reviewed the data. All authors contributed intellectually to the manuscript and approved the final version of the manuscript.

\section{Acknowledgements}

The Authors would like to acknowledge Drs John Cooper and Yun Tran, Renal Pathologists at the queen Elizabeth Hospital in Adelaide for providing the histology pictures.

\section{REFERENCES}

[1] S. P. McDonald and G. R. Russ, "Burden of End-Stage Renal Disease among Indigenous Peoples in Australia and New Zealand,” Kidney International, Vol. 63, 2003, pp. S123-S127. doi:10.1046/j.1523-1755.63.s83.26.x

[2] S. P. McDonald and G. R. Russ, "Current Incidence, Treatment Patterns and Outcome of End-Stage Renal Disease among Indigenous Groups in Australia and New Zealand,” Nephrology, Vol. 8, No. 1, 2003, pp. 42-48. doi:10.1046/j.1440-1797.2003.00131.x

[3] K. E. Yeates, A. Cass, T. D. Sequist, S. P. McDonald, M. J. Jardine, L. Trpeski, et al., "Indigenous People in Australia, Canada, New Zealand and the United States Are Less Likely to Receive Renal Transplantation,” Kidney International, Vol. 76, No. 6, 2009, pp. 659-664. doi:10.1038/ki.2009.236

[4] B. A. Pussell, A. Bendorf and I. H. Kerridge, “Access to the Kidney Transplant Waiting List: A Time for Reflection,” Internal Medicine Journal, Vol. 42, No. 4, 2012, pp. 360-363. doi:10.1111/j.1445-5994.2012.02730.x

[5] S. McDonald, "Indigenous Transplant Outcomes in Australia: What the ANZDATA Registry Tells Us," $\mathrm{Ne}$ phrology, Vol. 9, No. 4, 2004, pp. S138-S143. doi:10.1111/j.1440-1797.2004.00350.x

[6] N. M. Rogers, P. D. Lawton and M. D. Jose, "Kidney Transplant Outcomes in the Indigenous Population in the Northern Territory of Australia," Transplantation, Vol. 82, No. 7, 2006, pp. 882-886. doi:10.1097/01.tp.0000232439.88527.49 
[7] N. M. Rogers, P. D. Lawton and M. D. Jose, "Indigenous Australians and Living Kidney Donation,” The New England Journal of Medicine, Vol. 361, No. 15, 2009, pp. 1513-1516. doi:10.1056/NEJMc0905777

[8] J. S. Davis, B. J. Currie, D. A. Fisher, S. E. Huffam, N. M. Anstey, R. N. Price, V. L. Krause, N. Zweck, P. D. Lawton, P. L. Snelling and S. Selva-Nayagam, "Prevention of Opportunistic Infections in Immunosuppressed Patients in the Tropical Top End of the Northern Territory of Australia,” Communicable Diseases Intelligence, Vol. 27, No. 4, 2003, pp. 526-532.

[9] N. M. Anstey, I. Bastian, H. Dunckley and B. J. Currie, "Systemic Lupus Erythematosus (SLE): Different Prevalences in Different Populations of Australian Aborigines," Australian and New Zealand Journal of Medicine, Vol. 25, No. 6, 1995, pp. 736-737. doi:10.1111/j.1445-5994.1995.tb02863.x

[10] N. M. Anstey, I. Bastian, H. Dunckley and B. J. Currie, "Systemic Lupus Erythematosus in Australian Aborigines: High Prevalence, Morbidity and Mortality," Australian and New Zealand Journal of Medicine, Vol. 23, No. 1993, pp. 646-651. doi:10.1111/j.1445-5994.1993.tb04720.x

[11] D. M. Grennan and D. Bossingham, "Systemic Lupus Erythematosus (SLE): Different Prevalences in Different Populations of Australian Aboriginals," Australian and New Zealand Journal of Medicine, Vol. 25, No. 2, 1995, pp. 182-183. doi:10.1111/j.1445-5994.1995.tb02843.x

[12] K. Andreasyan and W. E. Hoy, "Patterns of Mortality in Indigenous Adults in the Northern Territory, 1998-2003: Are People Living in More Remote Areas Worse off?" The Medical Journal of Australia, Vol. 190, No. 6, 2009, pp. 307-311.

[13] K. Andreasyan and W. E. Hoy, "Recent Patterns in Chronic Disease Mortality in Remote Living Indigenous Australians,” BMC Public Health, Vol. 10, No. 1, 2010, pp. 483487. doi:10.1186/1471-2458-10-483

[14] N. M. Rogers, P. D. Lawton and M. D. Jose, "Plasma Cell Infiltrates and Renal Allograft Outcomes in Indigenous and Non-Indigenous People of the Northern Territory of
Australia,” Nephrology, Vol. 16, No. 8, 2011, pp. 777783.

[15] K. Anderson, A. Cass, J. Cunningham, P. Snelling, J. Devitt and C. Preece, "The Use of Psychosocial Criteria in Australian Patient Selection Guidelines for Kidney Transplantation,” Social Science \& Medicine, Vol. 64, No. 10, 2007, pp. 2107-2114.

[16] K. Anderson, J. Devitt, J. Cunningham, C. Preece, M. Jardine and A. Cass, "If You Can’t Comply with Dialysis, How Do You Expect Me to Trust You with Transplantation? Australian Nephrologists' Views on Indigenous Australians' 'Non-Compliance' and Their Suitability for Kidney Transplantation,” International Journal for Equity in Health, Vol. 11, 2012, p. 21. doi:10.1186/1475-9276-11-21

[17] A. Cass, J. Cunningham, K. Anderson, P. Snelling, S. A. M. Colman, J. Devitt, et al., "Decision-Making about Suitability for Kidney Transplantation: Results of a National Survey of Australian Nephrologists,” Nephrology, Vol. 12, No. 3, 2007, pp. 299-304. doi:10.1111/j.1440-1797.2007.00784.x

[18] A. Cass, J. Cunningham, P. Snelling, Z. Wang and W. Hoy, "Renal Transplantation for Indigenous Australians: Identifying the Barriers to Equitable Access,” Ethnicity \& Health, Vol. 8, No. 2, 2003, pp. 111-119. doi:10.1080/13557850303562

[19] A. Cass, J. Devitt, C. Preece, J. Cunningham, K. Anderson, P. Snelling, et al., "Barriers to Access by Indigenous Australians to Kidney Transplantation: The IMPAKT Study,” Nephrology, Vol. 9, Suppl. 4, 2004, pp. S144S146. doi:10.1111/j.1440-1797.2004.00352.x

[20] J. Devitt, A. Cass, J. Cunningham, C. Preece, K. Anderson and P. Snelling, "Study Protocol-Improving Access to Kidney Transplants (IMPAKT): A Detailed Account of a Qualitative Study Investigating Barriers to Transplant for Australian Indigenous People with End-Stage Kidney Disease,” BMC Health Services Research, Vol. 8, No. 1, 2008, pp. 24-31. doi:10.1186/1472-6963-8-31

\author{
Abbreviations \\ NR: Normal Reference Range \\ CMV: Cytomegalovirus \\ IMPAKT Study: The Improving Access to Kidney Trans- \\ plants Study \\ DNA: Deoxyribonucleic Acid
}

ANZDATA: Australia and New Zealand Dialysis and Transplant Registry 